\title{
Current Guidelines for Management of Medullary Thyroid Carcinoma
}

\author{
Mijin Kim, Bo Hyun Kim \\ Department of Internal Medicine, Biomedical Research Institute, Pusan National University Hospital, Busan, Korea
}

Medullary thyroid carcinoma (MTC) is a rare neuroendocrine tumor originating from the parafollicular cells. The diagnostic and therapeutic strategies for the condition are different from those used for well-differentiated thyroid cancer. Since the 2015 American Thyroid Association guidelines for the diagnosis and treatment of MTC, the latest, including the National Comprehensive Cancer Network and European Association for Medical Oncology guidelines have been updated to reflect several recent advances in the management of MTC. Advances in molecular diagnosis and postoperative risk stratification systems have led to individualized treatment and follow-up strategies. Multi-kinase inhibitors, such as vandetanib and cabozantinib, can prolong disease progression-free survival with favorable adverse effects. In addition, potent selective rearranged during transfection (RET) inhibitors (selpercatinib and pralsetinib) have shown a promising efficacy in recent clinical trials. This review summarizes the management of MTC in recent guidelines focused on sporadic MTC.

Keywords: Thyroid cancer, medullary; Cabozantinib; Pralsetinib; Selpercatinib; Guidelines; Precision medicine

\section{INTRODUCTION}

Medullary thyroid carcinoma (MTC) is a rare neuroendocrine tumor that can produce calcitonin from parafollicular cells. Thus, both the diagnostic and therapeutic strategies for MTC differed from those used for well-differentiated thyroid cancer (DTC) derived from follicular cells. MTC accounts for $0.6 \%$ of all thyroid cancers in Korea and $1 \%$ to $2 \%$ in the United States $[1,2]$. MTC occurs in a sporadic form and is associated with multiple endocrine neoplasia (MEN) type 2 as a hereditary form, accounting for $75 \%$ and $25 \%$, respectively [1].

Sporadic MTC is usually diagnosed as advanced disease in many patients because the most common presentation is a solitary thyroid nodule without any symptoms. The diagnosis of sporadic MTC is mostly based on fine-needle aspiration (FNA)

Received: 27 April 2021, Revised: 18 May 2021, Accepted: 31 May 2021

Corresponding author: Bo Hyun Kim

Department of Internal Medicine, Biomedical Research Institute, Pusan National University Hospital, 305 Gudeok-ro, Seo-gu, Busan 49241, Korea Tel: +82-51-240-7236, Fax: +82-51-254-3237, E-mail: pons71@hanmail.net biopsy and immunohistochemical staining for calcitonin [1]. However, preoperative diagnosis of MTC is difficult because of the presentation of a solitary thyroid nodule and its rare incidence. Therefore, MTC is sometimes diagnosed after thyroidectomy for indeterminate or suspicious malignant FNA results [1].

Complete surgical resection of the thyroid tumor and any locoregional metastases is the only curative option for locoregional MTC. Therefore, early diagnosis and detection of MTC are very important for prognosis [3]. Treatment options for recurrent or metastatic MTC include surgical resection, external beam radiation therapy (EBRT), and directed local therapies or systemic therapies [1,4]. However, the best management is still controversial in patients with recurrent MTC and distant metastases, because many patients with metastatic disease have indolent disease courses for a long time [1,4]. Management guide-

Copyright $\odot 2021$ Korean Endocrine Society

This is an Open Access article distributed under the terms of the Creative Commons Attribution Non-Commercial License (https://creativecommons.org/ licenses/by-nc/4.0/) which permits unrestricted non-commercial use, distribution, and reproduction in any medium, provided the original work is properly cited. 
lines for MTC have been published and updated internationally by several societies. Guidelines from the American Thyroid Association (ATA) were updated in 2015 [1]. Subsequently, treatment guidelines for MTC have been updated to reflect the results of recent research and clinical trials. The European Association for Medical Oncology (ESMO) clinical practice guidelines also provide updated recommendations on the diagnosis and treatment of MTC [4]. The Japan Associations of Endocrine Surgeons (JAES) has revised the clinical practice guidelines for thyroid tumors including MTC [5]. Lastly, the National Comprehensive Cancer Network (NCCN) clinical practice guidelines in oncology were updated (version 3.2020; February 2, 2021) [6]. This review summarizes the management of MTC in recent guidelines focused on sporadic MTC.

\section{PREOPERATIVE EVALUATION}

Serum calcitonin and carcinoembryonic antigen (CEA) in MTC are important diagnostic, prognostic, and predictive biomarkers because of their direct relationship with the C-cell mass [7]. If MTC is confirmed on FNA, thorough neck ultrasonography (US) should be performed to identify neck lymph node (LN) metastasis. In patients with extensive neck disease, serum calcitonin levels greater than $500 \mathrm{pg} / \mathrm{mL}$, signs of distant metastasis, contrast-enhanced (CE) computed tomography (CT) of the neck and chest, three-phase $\mathrm{CE}$ liver protocol CT or CE magnetic resonance imaging (MRI) of the liver, and bone scan and axial MRI are considered [1]. However, 2-[ $\left.{ }^{18} \mathrm{~F}\right]$-fluoro-2-deoxy-Dglucose (FDG)-positron emission tomography (PET/CT) or ${ }^{18} \mathrm{~F}$ dihydroxyphenyl-alanine (F-DOPA)-PET/CT is not recommended because they are less sensitive in detecting metastases [1]. NCCN guidelines also recommend that CE CT of the neck and chest, 3 phase liver protocol CT, liver MRI, bone scan and/ or skeletal MRI are indicated based on a high burden of disease, calcitonin above $400 \mathrm{pg} / \mathrm{mL}$, or elevated CEA levels [6]. If available, gallium-68-1,4,7,10-tetraazacyclododecane-1,4,7,10tetraacetic acid -octreotate (DOTATATE) PET/CT can be optionally considered in such patients because of the improved localization of MTC $[6,8]$. The ESMO guidelines recommend if F-DOPA-PET/CT were available in MTC patients with high serum calcitonin levels ( $\geq 500 \mathrm{pg} / \mathrm{mL}$ ) [4].

Analysis of germline rearranged during transfection (RET) mutations may be performed perioperatively. RET mutations are detected in all inherited MTC and present in $6 \%$ to $10 \%$ of apparent sporadic MTC [1]. Therefore, genetic testing for RET proto-oncogene mutations is recommended for all patients with clinically apparent sporadic MTC. It is also recommended for screening children and adults with hereditary MTC in known

Table 1. Incidence of Associated Tumors in Multiple Endocrine Neoplasia 1 and 2 According to Common RET Mutation Status ${ }^{\mathrm{a}}$

\begin{tabular}{|c|c|c|c|c|c|c|}
\hline RET mutation & Exon & $\begin{array}{c}\text { Risk of aggressive } \\
\text { MTC }\end{array}$ & Pheochromocytoma & $\begin{array}{c}\text { Primary } \\
\text { hyperparathyroidism }\end{array}$ & $\begin{array}{l}\text { Cutaneous lichen } \\
\text { amyloidosis }\end{array}$ & $\begin{array}{l}\text { Hirschsprung's } \\
\text { disease }\end{array}$ \\
\hline G553C & 8 & Moderate & $\sim 10 \%$ & - & $\mathrm{N}$ & $\mathrm{N}$ \\
\hline $\mathrm{C} 609 \mathrm{~F} / \mathrm{G} / \mathrm{R} / \mathrm{S} / \mathrm{Y}$ & 10 & Moderate & $\sim 10 \%-30 \%$ & $\sim 10 \%$ & $\mathrm{~N}$ & Y \\
\hline $\mathrm{C} 611 \mathrm{~F} / \mathrm{G} / \mathrm{S} / \mathrm{Y} / \mathrm{W}$ & 10 & Moderate & $\sim 10 \%-30 \%$ & $\sim 10 \%$ & $\mathrm{~N}$ & $\mathrm{Y}$ \\
\hline $\mathrm{C} 618 \mathrm{~F} / \mathrm{R} / \mathrm{S}$ & 10 & Moderate & $\sim 10 \%-30 \%$ & $\sim 10 \%$ & $\mathrm{~N}$ & $\mathrm{Y}$ \\
\hline $\mathrm{C} 620 \mathrm{~F} / \mathrm{R} / \mathrm{S}$ & 10 & Moderate & $\sim 10 \%-30 \%$ & $\sim 10 \%$ & $\mathrm{~N}$ & Y \\
\hline $\mathrm{C} 630 \mathrm{R} / \mathrm{Y}$ & 11 & Moderate & $\sim 10 \%-30 \%$ & $\sim 10 \%$ & $\mathrm{~N}$ & $\mathrm{~N}$ \\
\hline D631Y & 11 & Moderate & $\sim 50 \%$ & - & $\mathrm{N}$ & $\mathrm{N}$ \\
\hline $\mathrm{C} 634 \mathrm{~F} / \mathrm{G} / \mathrm{R} / \mathrm{S} / \mathrm{W} / \mathrm{Y}$ & 11 & High & $\sim 50 \%$ & $\sim 20 \%-30 \%$ & $\mathrm{Y}$ & $\mathrm{N}$ \\
\hline K666E & 11 & Moderate & $\sim 10 \%$ & - & $\mathrm{N}$ & $\mathrm{N}$ \\
\hline L790F & 13 & Moderate & $\sim 10 \%$ & - & $\mathrm{N}$ & $\mathrm{N}$ \\
\hline V804L & 14 & Moderate & $\sim 10 \%$ & $\sim 10 \%$ & $\mathrm{~N}$ & $\mathrm{~N}$ \\
\hline V804M & 14 & Moderate & $\sim 10 \%$ & $\sim 10 \%$ & Y & $\mathrm{N}$ \\
\hline A883F & 15 & High & $\sim 50 \%$ & - & $\mathrm{N}$ & $\mathrm{N}$ \\
\hline S891A & 15 & Moderate & $\sim 10 \%$ & $\sim 10 \%$ & $\mathrm{~N}$ & $\mathrm{~N}$ \\
\hline M918T & 16 & Highest & $\sim 50 \%$ & - & $\mathrm{N}$ & $\mathrm{N}$ \\
\hline
\end{tabular}

RET, rearranged during transfection; MTC, medullary thyroid carcinoma; $\mathrm{N}$, negative occurrence; $\mathrm{Y}$, positive occurrence.

${ }^{a}$ Modified by 2015 Revised American Thyroid Association guidelines for the management of medullary thyroid carcinoma. 
relatives $[1,4]$. Specific germline RET mutations in inherited MTC are closely associated with genotype-phenotype affecting the onset time of disease and tumor aggressiveness (Table 1) [1]. It is well known that RET M918T somatic mutation is associated with more aggressive features such as distant metastasis than wild-type MTC [9]. However, the ATA guidelines suggest that it is not standard practice to analyze RET M918T somatic mutations in patients with sporadic MTC [1]. The 2019 ESMO guidelines optionally recommended that somatic RET mutation testing may be needed for individual targeted therapy if selective RET inhibitor treatment is planned in patients with advanced MTCs [4].

Preoperative evaluation of coexisting pheochromocytoma is very important to prevent hypertensive crisis during surgery. Therefore, pheochromocytoma and primary hyperparathyroidism should be excluded in all patients with either an unknown RET mutation status or a germline RET mutation [1]. Biochemical evaluation, including serum calcium and plasma fractionated metanephrines, should be performed. If a pheochromocytoma coexists, it should be treated with alpha-blocker and removed by laparoscopic or retroperitoneal adrenalectomy before thyroid surgery to prevent hypertensive crisis $[1,4,6]$.

\section{SURGICAL MANAGEMENT}

As mentioned previously, complete surgical resection of the thyroid mass and locoregional metastasis is the only curative option for locoregional MTC. Total thyroidectomy is the preferred surgical approach because bilateral or multifocal disease is occurred in all patients with inherited MTC and approximately $10 \%$ of sporadic MTC $[1,4]$. Total thyroidectomy and dissection of cervical LN compartments is a standard approach for sporadic or inherited MTC according to preoperative serum calcitonin values, US findings, and intraoperative proven LN metastasis [1,4-6]. However, there is a controversial issue in performing lateral neck dissection (ND) in patients without evidence of LN metastasis on preoperative US. Ipsilateral ND is generally performed due to intraoperative evidence of central LN involvement. More aggressive prophylactic lateral ND can lead to postoperative morbidity without obvious survival benefits [1].

Prophylactic lateral ND for MTC without evidence of neck metastasis and distant metastasis could not achieve a consensus agreement in the ATA guidelines committee, but they recommend neither for nor against prophylactic lateral ND being considered based on serum calcitonin levels [1]. Total thyroidecto- my, central LN dissection, and the dissection involved lateral neck compartments (levels II-V) should be performed in restricted disease of the neck $[1,4,6]$. Contralateral ND should be considered if the basal serum calcitonin level is greater than 200 $\mathrm{pg} / \mathrm{mL}$ when the ipsilateral lateral neck $\mathrm{LN}$ is positive but the contralateral neck compartment is negative on preoperative imaging according to ATA and ESMO guidelines [1,4]. The ESMO guidelines generally recommended total thyroidectomy with bilateral central ND and ipsilateral ND at least at levels IIA, III, and IV even if serum calcitonin level is 50 to $200 \mathrm{pg} / \mathrm{mL}$ in patients with negative neck US [4]. There is no need to perform prophylactic central ND in small intrathyroidal MTC with a preoperative calcitonin level $<20 \mathrm{pg} / \mathrm{mL}$ because of no risk of LN metastasis $[1,4,10]$.

According to the NCCN guidelines, total thyroidectomy is recommended, and ND can be considered for unilateral thyroid disease with less than $1 \mathrm{~cm}$. Total thyroidectomy and bilateral central ND are indicated in all MTCs with bilateral thyroid disease or tumor size $\geq 1 \mathrm{~cm}$. More extensive lateral ND (levels II$\mathrm{V}$ ) is considered in patients with MEN2A and familial MTC in cases of tumors greater than $1 \mathrm{~cm}$ or central LN metastasis [6]. Even if the MTC is locally advanced or metastatic, total thyroidectomy with involved LN compartments dissection is recommended to preserve the function of speech, swallowing, and the parathyroid gland [1]. It is very important that individualized surgical decisions should be made according to life expectancy, underlying comorbidities, and the patient's wishes.

\section{POSTOPERATIVE MANAGEMENT}

If MTC is postoperatively diagnosed after lobectomy, completion thyroidectomy is not routinely performed in patients without a germline RET mutation. However, completion thyroidectomy is indicated in sporadic MTC with detectable postoperative serum calcitonin and abnormal neck US [1,4]. In cases of inadequate lateral ND at the initial surgery, compartment-oriented lateral ND should be performed if the preoperative serum calcitonin level is less than $1,000 \mathrm{pg} / \mathrm{mL}$ and five or fewer metastatic LNs are removed at the initial operation based on an expert opinion of the ATA guidelines [1].

The eighth edition of the American Joint Committee on Cancer/Union for International Cancer Control (AJCC/UICC) staging system for thyroid cancer was published in late 2016. There were some major changes, including a new definition of T3 classification (removal of minor extrathyroidal extension from $\mathrm{T} 3$ disease) and an increase in the cutoff age (55 years) for high 
risk in DTC. The AJCC/UICC tumor node metastasis (TNM) staging system is also recommended for MTC [11]. However, a TNM staging system based on DTC cannot be applied uniformly to the MTC. The eighth AJCC/UICC staging system for MTC does not seems to be the best in differentiating mortality risk among stage groups and upstaging many patients to stage IV [12]. Therefore, the NCCN guidelines do not use TNM stages as the initial determinant of management because of the lack of other important prognostic factors, such as age of patients at diagnosis, especially in inherited MTC and postoperative serum calcitonin levels [6].

Postoperative management depends on postoperative serum calcitonin and CEA levels to detect residual diseases (Fig. 1).
Because serum calcitonin slowly decreases in MTC patients, the nadir of calcitonin level cannot be reached for several months. Therefore, the timing of measurement of serum calcitonin level after surgery is crucial [13]. ATA guidelines recommend that serum calcitonin and CEA levels should be measured 3 months postoperatively [1]. The ESMO and NCCN guidelines also recommend that they be measured 30 to 60 days and 2 or 3 months after surgery, respectively [4,6]. Postoperative disease monitoring depends on serum calcitonin and CEA levels in the ATA and NCCN guidelines, and ESMO guidelines suggest dynamic risk stratification (Fig. 1) [4]. As dynamic risk stratification has been used with clinical values in DTC, dynamic risk stratification for MTC was developed to modify initial risk esti-

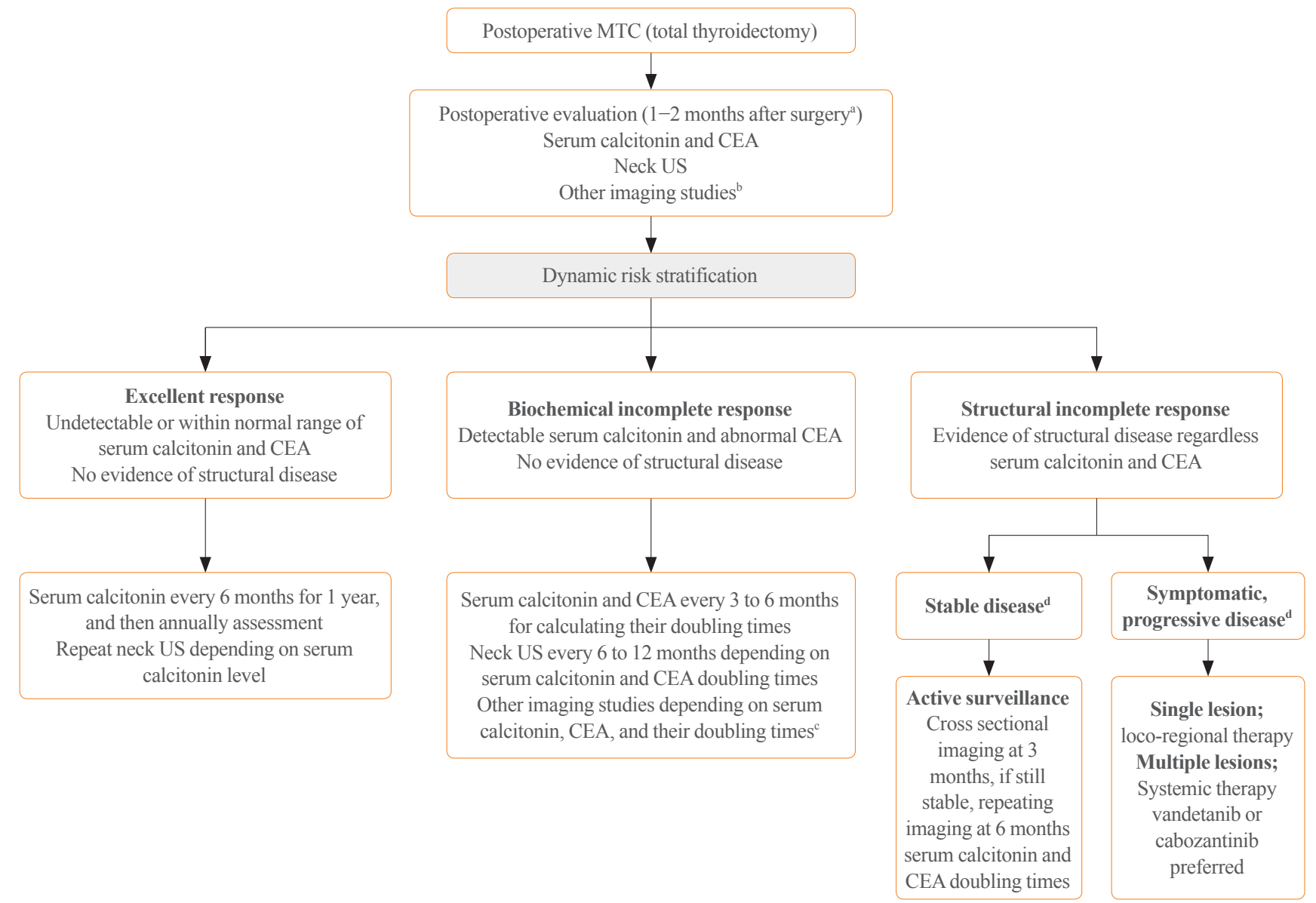

Fig. 1. Postoperative management in patients with medullary thyroid cancer (MTC) according to dynamic risk stratification in European Association for Medical Oncology (ESMO) guidelines. CEA, carcinoembryonic antigen; US, ultrasonography. ${ }^{a}$ American Thyroid Association (ATA) guidelines recommend that serum calcitonin and CEA should be measured after 3 months postoperatively; ${ }^{\mathrm{b}}$ Other imaging modalities including contrast-enhanced (CE) computed tomography (CT) of neck, chest, abdomen with liver protocol, and bone scan, axial magnetic resonance imaging (MRI) depending on the tumor stage, serum calcitonin ( $\geq 150 \mathrm{pg} / \mathrm{mL})$ and CEA; ${ }^{\circ}$ Negative other imaging modalities, consider $\left[{ }^{18} \mathrm{~F}\right]$-fluoro-2-deoxy-D-glucose (F18-FDG) positron emission tomography-CT or Gallium-68 DOTATATE or CE MRI with neck,

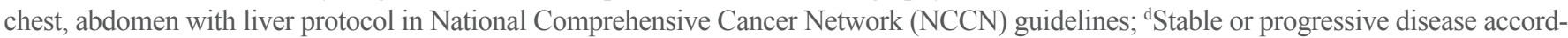
ing to Response Evaluation Criteria in Solid Tumors (RECIST) version 1.1. 
mation based on the response to therapy and biological tumor behavior, including change in serum calcitonin and CEA levels, and it has provided more useful real-time prognostic information than initial TNM staging [14-16].

Postoperative persistent hypercalcitoninemia, short doubling times of calcitonin and CEA ( $<6$ to 12 months), and rapid increase in CEA levels in a stable state of calcitonin level may be other important prognostic factors $[1,4,6]$. Codon analysis of $R E T$ proto-oncogene mutations is also useful for predicting prognosis because specific germline RET mutations predict tumor aggressiveness in hereditary MTC $[1,4,6]$. In addition, a tumor volume doubling time of less than 1 year in MTC patients with lung metastases is correlated with poorer overall survival [17].

Postoperative adjuvant EBRT to the neck and mediastinum should be considered in patients at high risk for airway obstruction and local recurrence, such as residual disease, extensive LN metastases, or extra-thyroidal extension as an expert opinion of ATA guidelines [1].

\section{MANAGEMENT IN PERSISTENT OR RECURRENT MTC}

Management options for recurrent or residual MTC include close observation for indolent disease, surgical resection of locoregional disease, EBRT, and local therapies, such as radiofrequency ablation, cryoablation, and embolization, or systemic therapies, such as conventional chemotherapy, kinase inhibitors, and immune checkpoint inhibitors for non-resectable diseases. Therefore, treatment approaches in these patients depend on various clinical factors, including presence of the symptoms, possibility of significant structural disease progression, disease localization, disease volume or burden, and location of the metastatic lesion $[1,4,6]$.

Patients with biochemical incomplete responses without structural disease are followed-up with conservative surveillance $[4,6]$. Therapeutic interventions based on increased biochemical markers are not recommended. In cases of locoregional disease without distant metastasis, surgical resection is the primary treatment option with or without postoperative EBRT or intensity-modulated radiation therapy [4,6]. However, active surveillance can be an optimal management modality in many patients with asymptomatic small LN metastases because repeat operations are commonly not curative and can be associated with high surgical complications, such as adjacent nerve injury and permanent hypoparathyroidism [1].
Currently, most guidelines do not recommend the use of single or combinational chemotherapeutic regimens as first-line systemic therapy $[1,4,6]$. Systemic therapy as a first-line regimen, such as vandetanib or cabozantinib, should be considered in unresectable locoregional diseases and distant metastases with symptomatic or progressive MTCs by Response Evaluation Criteria in Solid Tumors (RECIST) [1,4,6,18]. Vandetanib and cabozantinib increased progression-free survival (PFS) in a phase III trial named the Zactima Efficacy in Thyroid Cancer Assessment (ZETA) trial [19] and Efficacy of XL184 (cabozantinib) in Advanced Medullary Thyroid Cancer (EXAM) trial [20], respectively (Table 2). Recent post hoc analysis from the ZETA trial also demonstrated that vandetanib treatment had significant prolonged median PFS (hazard ratio [HR], 0.43; 95\% confidence interval $[\mathrm{CI}], 0.28$ to $0.64 ; P<0.001)$ in patients with symptomatic and progressive diseases [21]. However, OS had not significant difference between vandetanib and placebo arm because of post-progression, unblinded, open-label treatment with vandetanib (HR, 1.08; 95\% CI, 0.72 to $1.61 ; P=0.71)$ [21].

Cabozantinib had a statistically non-significant increase in median OS compared with placebo (26.6 months for cabozantinib vs. 21.1 months for placebo: HR, $0.85 ; 95 \% \mathrm{CI}, 0.64$ to $1.12 ; P=0.24)$ [22]. However, cabozantinib treatment had a significant benefit in patients with RET M918T-positive MTC (median OS 44.3 months for cabozantinib vs. 18.9 months for placebo: HR, $0.60 ; 95 \%$ CI, 0.38 to $0.94 ; P=0.03$ ) [22].

Several recent real-world multicenter studies and meta-analysis also showed that vandetanib and cabozantinib had significant clinical benefits for objective response rate (ORR) and PFS in advanced MTC [23-26]. We demonstrated that median PFS from the start of vandetanib was 25.9 months, but the median OS was not reached [23]. In addition, German real world multicenter study recently reported that the median OS from the initiation of vandetanib therapy was 53 months (95\% CI, 43.7 to 62.3 ) and 24 months ( $95 \%$ CI, 5.9 to 42.1 ) for cabozantinib [24].

Unfortunately, there is no clear evidence as to which regimen between vandetanib and cabozantinib is better. Although cabozantinib seems to be effective as a second-line therapy [22], the choice of the first regimen among vandetanib and cabozantinib should be considered for potential toxicity in each patient. However, cabozantinib has not yet been approved for the treatment of MTC in South Korea.

The NCCN panels also recommend that other tyrosine kinase inhibitors (TKIs), including sunitinib, lenvatinib, sorafenib, and pazopanib are considered if clinical trials of vandetanib or cabo- 
Table 2. Summary of Phase 3 Clinical Trials of Vandetanib or Cabozantinib versus Placebo in Patients with Advanced Medullary Thyroid Cancer

\begin{tabular}{|c|c|c|}
\hline Variable & Vandetanib (300 mg/day) & Cabozantinib (140 mg/day) \\
\hline Targets & VEGFR, RET, EGFR, c-KIT & VEGFR, RET, c-MET, c-KIT \\
\hline Phase 3 clinical trial & ZETA study & EXAM study \\
\hline No. of patients (mean age, years) & Vandetanib 231 (50.7) vs. Placebo 100 (53.4) & Cabozantinib 219 (55.0) vs. Placebo 111 (55.0) \\
\hline Postprogression, open-label treatment & Yes & No \\
\hline Radiologic progression before enrolment & Not requested & Yes (within 14 mo) \\
\hline Previous treatment & $40 \%$ & $38 \%$ \\
\hline Previous TKIs & Unknown & $20 \%$ \\
\hline Hereditary disease & $10 \%$ & $5.5 \%$ \\
\hline RET mutation positive & $59 \%$ Vandetanib arm & $46.1 \%$ Cabozantinib arm \\
\hline RET 918 mutation positive & Not available & $34.2 \%$ Cabozantinib arm \\
\hline Median time of follow-up, mo & 24 & 13.9 \\
\hline \multicolumn{3}{|l|}{ Results } \\
\hline Median progression-free survival, mo & $\begin{array}{l}30.5 \text { vs. } 19.3 \text { (HR, } 0.46 ; 95 \% \text { CI, } 0.31-0.69 \text {; } \\
\quad P<0.001)\end{array}$ & $\begin{array}{l}11.2 \text { vs. } 4.0(\mathrm{HR}, 0.28 ; 95 \% \mathrm{CI}, 0.19-0.40 \\
P<0.001)\end{array}$ \\
\hline Objective response rate & $45 \%$ vs. $13 \%(P<0.001)$ & $28 \%$ vs. $0 \%(P<0.001)$ \\
\hline Overall survival, mo & Not available & $\begin{array}{l}26.6 \text { vs. } 21.1 \text { (HR, } 0.85 ; 95 \% \text { CI, } 0.64-1.12 \text {; } \\
\quad P=0.24)\end{array}$ \\
\hline Overall survival in RET positive, mo & Not available & $\begin{array}{l}44.3 \text { vs. } 18.9 \text { (HR, } 0.60 ; 95 \% \text { CI, } 0.38-0.94 \text {; } \\
\qquad P=0.03)\end{array}$ \\
\hline \multicolumn{3}{|l|}{ Safety } \\
\hline Most common adverse events at least grade 3 & $\begin{array}{l}\text { Diarrhea, hypertension, QTc prolongation, } \\
\text { fatigue }\end{array}$ & $\begin{array}{l}\text { Diarrhea, palmar-plantar erythrodysesthesia, } \\
\text { fatigue }\end{array}$ \\
\hline
\end{tabular}

VEGFR, vascular endothelial growth factor receptor; RET, rearranged during transfection; EGFR, epidermal growth factor receptor; KIT, v-kit HardyZuckerman 4 feline sarcoma viral oncogene; c-MET, hepatocyte growth factor receptor; ZETA, Zactima Efficacy in Thyroid Cancer Assessment; EXAM, Efficacy of XL184 (cabozantinib) in Advanced Medullary Thyroid Cancer; TKI, tyrosine kinase inhibitor; HR, hazard ratio; CI, confidence interval; QTc, corrected QT.

zantinib are not available [6]. Among these kinase inhibitors, sunitinib and lenvatinib have good response rates [27-29]. If the disease progresses despite vandetanib or cabozantinib treatment, dacarbazine or combination chemotherapy can be administered [6,30]. Clinical studies for other novel multi-targeted TKIs, such as ponatinib (AP24534), anlotinib (AL3818), and TPX-0046, are ongoing in advanced or metastatic MTC [3133].

The latest NCCN guidelines for MTC management introduced highly selective RET inhibitors, including selpercatinib (LOXO-292) and pralsetinib (BLU-667) for the first time [6]. They have higher potency and lower toxicity. Selpercatinib and pralsetinib can be considered for patients with a positive $R E T$ somatic mutation [6].

Selpercatinib is a highly selective small molecule RET kinase inhibitor that has very high potency to inhibit different $R E T$ al- terations, both point mutations and fusions, including the RET V804M mutation responsible for other TKI resistance [34]. Selpercatinib (160 mg twice daily) showed remarkable and durable efficacy in MTC with or without prior vandetanib or cabozantinib treatment in phase $1 / 2$ trials (LIBRETTO-001 trial) (Table 3) [34]. The ORR was $69 \%(95 \%$ CI, 55 to 81$)$ and 1-year PFS was $82 \%$ (95\% CI, 69 to 90) in patients with RETmutant MTC who had previously been treated with vandetanib, cabozantinib, or both. The ORR was $73 \%$ (95\% CI, 62 to 82 ) and 1-year PFS was $92 \%(95 \%$ CI, 82 to 97$)$ in patients with RET-mutant MTC without previous treatment with vandetanib or cabozantinib. The most common severe adverse events (grade 3 or 4) were hypertension, increased liver enzymes, hyponatremia, and diarrhea [34]. Recently, the U.S. Food and Drug Administration approved the use of selpercatinib in patients with advanced or metastatic RET-mutant MTC [35]. A 
Table 3. Summary of Clinical Trials of Selpercatinib and Pralsetinib versus Placebo in Patients with Advanced MTC with RET Alteration

\begin{tabular}{|c|c|c|c|c|}
\hline \multirow[b]{2}{*}{ Variable } & \multicolumn{2}{|c|}{ Selpercatinib } & \multicolumn{2}{|c|}{ Pralsetinib $^{\mathrm{a}}$} \\
\hline & $\begin{array}{l}\text { RET-mutant MTC with } \\
\text { prior TKI }(n=55)\end{array}$ & $\begin{array}{c}R E T \text {-mutant MTC } \\
\text { without prior TKI }(n=88)\end{array}$ & $\begin{array}{l}\text { RET-mutant MTC with } \\
\text { prior TKI }(n=61)\end{array}$ & $\begin{array}{c}\text { RET-mutant MTC } \\
\text { without prior TKI }(n=22)\end{array}$ \\
\hline
\end{tabular}

\begin{tabular}{|c|c|c|c|c|}
\hline Clinical trial name & \multicolumn{2}{|c|}{ LIBRETTO-001 (phase 1/2) } & \multicolumn{2}{|c|}{ ARROW (phase 1/2) } \\
\hline Dosage (Phase 2) & \multicolumn{2}{|c|}{$160 \mathrm{mg}$ twice daily } & \multicolumn{2}{|c|}{$400 \mathrm{mg}$ once daily } \\
\hline Median age, yr (range) & $57(17-84)$ & $58(15-82)$ & $58(25-83)$ & $60(19-81)$ \\
\hline Male sex & $36(65)$ & $58(66)$ & $41(67)$ & $16(73)$ \\
\hline
\end{tabular}

Previous regimen

$\begin{array}{lll}\text { Vandetanib } & 18(33) & 0 \\ \text { Cabozantinib } & 13(24) & 0 \\ \text { Vandetanib and cabozantinib } & 24(44) & 0\end{array}$

Vandetanib and/or cabozantinib

$61(100)$

RET alteration

RET M918T mutation

RET V804M/L mutation

RET extracellular cysteine mutation ${ }^{\mathrm{b}}$

Other mutations $^{c}$

Objective response, $\%(95 \% \mathrm{CI})$

Complete response

Partial response

Stable disease

Progressive disease

Duration of response

Median, mo (95\% CI)

Median follow-up, mo

Progression-free survival

Median, mo $(95 \% \mathrm{CI})$

Median follow-up, mo

Disease control rate, $\%(95 \% \mathrm{CI})$

$33(60)$

5 (9)

$7(13)$

$10(18)$

$69(55-81)$

$5(9)$

$33(60)$

$14(25)$

$1(2)$

NE (19.1-NE)

14.1

NE (24.4-NE)

16.7

94

Values are expressed as number (\%) unless otherwise indicated.

RET, rearranged during transfection; MTC, medullary thyroid carcinoma; TKI, tyrosine kinase inhibitor; LIBRETTO-001, A Study of LOXO-292 in Participants With Advanced Solid Tumors, RET Fusion-Positive Solid Tumors, and Medullary Thyroid Cancer; ARROW, Phase 1/2 Study of the Highly-selective RET Inhibitor, Pralsetinib (BLU-667), in Patients With Thyroid Cancer, Non-Small Cell Lung Cancer, and Other Advanced Solid Tumors; CI, confidence interval; NE, not estimated; NR, not reached.

${ }^{a}$ Blinded independent central review of tumor response in response-evaluable patients enrolled by July 11, 2019, as of a data cutoff February 13.2020; ${ }^{b} \mathrm{Ex}-$ tracellular cysteine mutation was defined as a mutation that included at least one of the following cysteine residues: 609, 611, 618, 620, 630, or 634; ${ }^{\circ}$ Other mutations included D631-liter633delinsE, E632-liter633del, A883F, D631-liter633delinsV, L790F, D898-E901del, D898-E901del+D903-S904delinsEP, K666 N, T636-V637insCRT, and D378-G385delinsE; ' The median is unstable because it is based on less than 10\% of the total number of events. multicenter, randomized, open-label, phase 3 trial (LIBRETTO-531) is ongoing to compare selpercatinib to cabozantinib or vandetanib in patients without prior kinase inhibitor therapy in RET-mutant MTC (NCT04211337).

On December 1, 2020, pralsetinib was approved for systemic therapy in advanced or metastatic RET mutation-positive MTC based on preliminary results of a phase $1 / 2$ study (ARROW trial, NCT03037385). Pralsetinib therapy (400 mg orally once daily) had an ORR of $60 \%$ (95\% CI, 46 to 74 ) in 55 patients with advanced or metastatic RET-mutant MTC who received 
prior therapy with vandetanib or cabozantinib. The ORR was $74 \%$ (95\% CI, 49 to 91) in 22 patients with RET-mutant MTC who did not receive prior therapy of vandetanib or cabozantinib (Table 3). The most common serious laboratory abnormalities (grade 3 or $4, \geq 2 \%$ ) were leukopenia, anemia, decreased phosphate, hypocalcemia, hyponatremia, increased liver enzymes, thrombocytopenia, and increased alkaline phosphatase levels (NCT03037385) [36,37]. A multicenter, randomized, open-label, phase 3 trial (AcceleRET-MTC) has recently begun to compare pralsetinib with cabozantinib or vandetanib in patients without prior multi-kinase inhibitor therapy in RET-mutant MTC (NCT04760288). Other highly selective RET inhibitors, such as TPX-0046 and BOS172738 are also being tested in clinical trials $[38,39]$.

Although the role of immunotherapy appears to be more promising in anaplastic thyroid cancer, to date, no immunotherapy has been approved for advanced MTC. It is well known that high tissue tumor mutational burden (TMB) could be a useful marker to recognize patients with recurrent or metastatic advanced solid tumors who will have a better therapeutic benefit from immune checkpoint inhibitors, such as pembrolizumab [40]. Therefore, the NCCN guidelines for MTC management suggest that pembrolizumab should be considered in patients with symptomatic, progressive MTC with high TMB ( $\geq 10 \mathrm{mu}-$ tations/megabase) [6]. Pembrolizumab (NCT03072160, NCT02721732), nivolumab (NCT03246958), and ipilimumab (NCT03246958) are currently being evaluated in phase 2 trials for the treatment of recurrent or metastatic MTC [41].

Various other investigational therapeutic modalities, including immunotherapy, tumor vaccines $[42,43]$, radio-immunotherapy using radiolabeled anti-CEA monoclonal antibodies [44, 45], and peptide receptor radionuclide therapy (PRRT) using ${ }^{177} \mathrm{Lu}$-labeled or ${ }^{90} \mathrm{Y}$-labeled somatostatin analogues $[46,47]$, have been developed.

Treatment with yttrium-90-DOTA-[D-Phe1-Tyr3]-octreotide $\left({ }^{90} \mathrm{Y}\right.$-DOTATOC) decreased serum calcitonin levels as a primary endpoint, and survival benefits in responders were reported in a phase 2 trial of metastatic MTC [47]. PRRT using ${ }^{177} \mathrm{Lu}$-octreotate could be a therapeutic option in MTC patients with a high tumor uptake on ${ }^{111}$ In-diethylenetriamine pentaacetic acid (DTPA)-octreotide scan and tumor somatostatin receptor type 2a (SSTR2a) receptor expression by immunohistochemistry [48]. The ESMO guidelines optionally recommend the use of radionuclide therapy in selected cases [4].

\section{CONCLUSIONS}

Since the ATA guidelines were published in 2015, treatment guidelines for MTC have been updated to reflect the results of recent research and novel conceptions. Extensive careful surgery is the only curative option for localized MTC. However, most repeated surgeries cannot improve the cure rate in patients with widespread locoregional or metastatic MTCs; thus, less aggressive management, including active surveillance, local therapy, and palliative therapy, should be considered first. Vandetanib or cabozantinib should be primarily considered in patients with non-resectable symptomatic, rapidly progressive MTC; they can extend PFS and provide long-term disease stabilization in some patients. However, complete responses by multi-kinase inhibitors are virtually uncommon and have many significant adverse effects due to off-target effects, which can lead to poor quality of life. Selpercatinib and pralsetinib have shown remarkable efficacy, including a few complete responses and favorable safety in phase $1 / 2$ trials. Thus, molecular testing for germline or somatic RET mutations will be essential for finding an appropriate candidate who may benefit from these RET inhibitors. Pembrolizumab has some promising results in advanced MTC but limited clinical application.

Despite recent advances in the management of advanced MTC, various unmet needs still exist. Further studies are required to determine the mechanism of resistance to novel TKIs, selective RET inhibitors, and immune checkpoint inhibitors and to identify the appropriate combination regimens with various therapies. Ultimately, an evidence-based individualized approach based on molecular stratification seems to be the best option in precision medicine for the management of MTC.

\section{CONFLICTS OF INTEREST}

No potential conflict of interest relevant to this article was reported.

\section{ACKNOWLEDGMENTS}

This research was supported by a clinical research grant from Pusan National University Hospital in 2021.

\section{ORCID}

Mijin Kim https://orcid.org/0000-0002-1538-8859

Bo Hyun Kim https://orcid.org/0000-0001-9632-9457 


\section{REFERENCES}

1. Wells SA Jr, Asa SL, Dralle H, Elisei R, Evans DB, Gagel RF, et al. Revised American Thyroid Association guidelines for the management of medullary thyroid carcinoma. Thyroid 2015;25:567-610.

2. Oh CM, Jung KW, Won YJ, Shin A, Kong HJ, Lee JS. Ageperiod-cohort analysis of thyroid cancer incidence in Korea. Cancer Res Treat 2015;47:362-9.

3. Pacini F, Castagna MG, Cipri C, Schlumberger M. Medullary thyroid carcinoma. Clin Oncol (R Coll Radiol) 2010;22: 475-85.

4. Filetti S, Durante C, Hartl D, Leboulleux S, Locati LD, Newbold K, et al. Thyroid cancer: ESMO clinical practice guidelines for diagnosis, treatment and follow-up. Ann Oncol 2019;30:1856-83.

5. Ito Y, Onoda N, Okamoto T. The revised clinical practice guidelines on the management of thyroid tumors by the Japan Associations of Endocrine Surgeons: core questions and recommendations for treatments of thyroid cancer. Endocr J 2020;67:669-717.

6. National Comprehensive Cancer Network. NCCN clinical practice guidelines in oncology: thyroid carcinoma [Internet]. Plymouth Meeting: NCCN; c2021 [cited 2021 Jun 4]. Available from: http://www.nccn.org/professionals/physician_gls/ pdf/thyroid.pdf.

7. Laure Giraudet A, Al Ghulzan A, Auperin A, Leboulleux S, Chehboun A, Troalen F, et al. Progression of medullary thyroid carcinoma: assessment with calcitonin and carcinoembryonic antigen doubling times. Eur J Endocrinol 2008; 158:239-46.

8. Tran K, Khan S, Taghizadehasl M, Palazzo F, Frilling A, Todd JF, et al. Gallium-68 dotatate PET/CT is superior to other imaging modalities in the detection of medullary carcinoma of the thyroid in the presence of high serum calcitonin. Hell J Nucl Med 2015;18:19-24.

9. Romei C, Casella F, Tacito A, Bottici V, Valerio L, Viola D, et al. New insights in the molecular signature of advanced medullary thyroid cancer: evidence of a bad outcome of cases with double RET mutations. J Med Genet 2016;53: 729-34.

10. Machens A, Dralle H. Biomarker-based risk stratification for previously untreated medullary thyroid cancer. J Clin Endocrinol Metab 2010;95:2655-63.

11. Amin MB, Edge S, Greene F, Byrd DR, Brookland RK, Washington MK, et al. 2017 AJCC cancer staging manual. 8th ed. New York: Springer; 2017.

12. Adam MA, Thomas S, Roman SA, Hyslop T, Sosa JA. Rethinking the current American Joint Committee on Cancer TNM staging system for medullary thyroid cancer. JAMA Surg 2017;152:869-76.

13. Stepanas AV, Samaan NA, Hill CS Jr, Hickey RC. Medullary thyroid carcinoma: importance of serial serum calcitonin measurement. Cancer 1979;43:825-37.

14. Lindsey SC, Ganly I, Palmer F, Tuttle RM. Response to initial therapy predicts clinical outcomes in medullary thyroid cancer. Thyroid 2015;25:242-9.

15. Kwon H, Kim WG, Jeon MJ, Song DE, Lee YM, Sung TY, et al. Dynamic risk stratification for medullary thyroid cancer according to the response to initial therapy. Endocrine 2016;53:174-81.

16. Krajewska J, Chmielik E, Jarzab B. Dynamic risk stratification in the follow-up of thyroid cancer: what is still to be discovered in 2017? Endocr Relat Cancer 2017;24:R387402.

17. Yeh T, Yeung M, Sherman EJ, Tuttle RM, Sabra MM. Structural doubling time predicts overall survival in patients with medullary thyroid cancer in patients with rapidly progressive metastatic medullary thyroid cancer treated with molecular targeted therapies. Thyroid 2020;30:1112-9.

18. Eisenhauer EA, Therasse P, Bogaerts J, Schwartz LH, Sargent D, Ford R, et al. New response evaluation criteria in solid tumours: revised RECIST guideline (version 1.1). Eur J Cancer 2009;45:228-47.

19. Wells SA Jr, Robinson BG, Gagel RF, Dralle H, Fagin JA, Santoro M, et al. Vandetanib in patients with locally advanced or metastatic medullary thyroid cancer: a randomized, double-blind phase III trial. J Clin Oncol 2012;30:13441.

20. Elisei R, Schlumberger MJ, Muller SP, Schoffski P, Brose MS, Shah MH, et al. Cabozantinib in progressive medullary thyroid cancer. J Clin Oncol 2013;31:3639-46.

21. Kreissl MC, Bastholt L, Elisei R, Haddad R, Hauch O, Jarzab B, et al. Efficacy and safety of vandetanib in progressive and symptomatic medullary thyroid cancer: post hoc analysis from the ZETA Trial. J Clin Oncol 2020;38:2773-81.

22. Schlumberger M, Elisei R, Muller S, Schoffski P, Brose M, Shah M, et al. Overall survival analysis of EXAM, a phase III trial of cabozantinib in patients with radiographically progressive medullary thyroid carcinoma. Ann Oncol 2017; 28:2813-9.

23. Kim M, Yoon JH, Ahn J, Jeon MJ, Kim HK, Lim DJ, et al. 
Vandetanib for the management of advanced medullary thyroid cancer: a real-world multicenter experience. Endocrinol Metab (Seoul) 2020;35:587-94.

24. Koehler VF, Adam P, Frank-Raue K, Raue F, Berg E, Hoster E, et al. Real-world efficacy and safety of cabozantinib and vandetanib in advanced medullary thyroid cancer. Thyroid 2021;31:459-69.

25. Ramos HE, Hecht F, Berdelou A, Borget I, Leboulleux S, Baudin E, et al. Long-term follow-up and safety of vandetanib for advanced medullary thyroid cancer. Endocrine 2021;71:434-42.

26. Efstathiadou ZA, Tsentidis C, Bargiota A, Daraki V, Kotsa K, Ntali G, et al. Benefits and limitations of TKIs in patients with medullary thyroid cancer: a systematic review and meta-analysis. Eur Thyroid J 2021;10:125-39.

27. Ravaud A, de la Fouchardiere C, Caron P, Doussau A, Do Cao C, Asselineau J, et al. A multicenter phase II study of sunitinib in patients with locally advanced or metastatic differentiated, anaplastic or medullary thyroid carcinomas: mature data from the THYSU study. Eur J Cancer 2017;76: 110-7.

28. Schlumberger M, Jarzab B, Cabanillas ME, Robinson B, Pacini F, Ball DW, et al. A phase II trial of the multitargeted tyrosine kinase inhibitor lenvatinib (E7080) in advanced medullary thyroid cancer. Clin Cancer Res 2016;22:44-53.

29. Hadoux J, Schlumberger M. Chemotherapy and tyrosine-kinase inhibitors for medullary thyroid cancer. Best Pract Res Clin Endocrinol Metab 2017;31:335-47.

30. Orlandi F, Caraci P, Berruti A, Puligheddu B, Pivano G, Dogliotti L, et al. Chemotherapy with dacarbazine and 5-fluorouracil in advanced medullary thyroid cancer. Ann Oncol 1994;5:763-5.

31. Li D, Chi Y, Chen X, Ge M, Zhang Y, Guo Z, et al. Anlotinib in locally advanced or metastatic medullary thyroid carcinoma: a randomized, double-blind phase IIB trial. Clin Cancer Res 2021 Apr 8 [Epub]. https://doi.org/10.1158/1078-0432. CCR-20-2950.

32. De Falco V, Buonocore P, Muthu M, Torregrossa L, Basolo F, Billaud M, et al. Ponatinib (AP24534) is a novel potent inhibitor of oncogenic RET mutants associated with thyroid cancer. J Clin Endocrinol Metab 2013;98:E811-9.

33. Drilon AE, Zhai D, Rogers E, Deng W, Zhang X, Ung J, et al. The next-generation RET inhibitor TPX-0046 is active in drug-resistant and naïve RET-driven cancer models. J Clin Oncol 2020;38(15 Suppl):3616.

34. Wirth LJ, Sherman E, Robinson B, Solomon B, Kang H,
Lorch J, et al. Efficacy of selpercatinib in RET-altered thyroid cancers. N Engl J Med 2020;383:825-35.

35. Bradford D, Larkins E, Mushti SL, Rodriguez L, Skinner AM, Helms WS, et al. FDA approval summary: selpercatinib for the treatment of lung and thyroid cancers with RET gene mutations or fusions. Clin Cancer Res 2021;27:21305.

36. Subbiah V, Hu MI, Ganior JF, Mansfield AS, Alonso G, Taylor MH, et al. Clinical activity of the RET inhibitor pralsetinib (BLU-667) in patients with RET fusion+ solid tumors. J Clin Oncol 2020;38(15 Suppl):109.

37. Markham A. Pralsetinib: first approval. Drugs 2020;80: 1865-70.

38. Drilon A, Rogers E, Zhai D, Deng W, Zhang X, Lee D, et al. 506P: TPX-0046 is a novel and potent RET/SRC inhibitor for RET-driven cancers. Ann Oncol 2019;30 Suppl 5:v190-1.

39. Schoffski P, Aftimos PG, Massard C, Italiano A, Jungels C, Andreas K, et al. A phase I study of BOS172738 in patients with advanced solid tumors with RET gene alterations including non-small cell lung cancer and medullary thyroid cancer. J Clin Oncol 2019;37(15 Suppl):TPS3162.

40. Marabelle A, Fakih M, Lopez J, Shah M, Shapira-Frommer R, Nakagawa K, et al. Association of tumour mutational burden with outcomes in patients with advanced solid tumours treated with pembrolizumab: prospective biomarker analysis of the multicohort, open-label, phase 2 KEYNOTE-158 study. Lancet Oncol 2020;21:1353-65.

41. Araque KA, Gubbi S, Klubo-Gwiezdzinska J. Updates on the management of thyroid cancer. Horm Metab Res 2020; 52:562-77.

42. Stift A, Sachet M, Yagubian R, Bittermann C, Dubsky P, Brostjan $C$, et al. Dendritic cell vaccination in medullary thyroid carcinoma. Clin Cancer Res 2004;10:2944-53.

43. Papewalis C, Wuttke M, Jacobs B, Domberg J, Willenberg $\mathrm{H}$, Baehring T, et al. Dendritic cell vaccination induces tumor epitope-specific Th1 immune response in medullary thyroid carcinoma. Horm Metab Res 2008;40:108-16.

44. Kraeber-Bodere F, Rousseau C, Bodet-Milin C, Ferrer L, Faivre-Chauvet A, Campion L, et al. Targeting, toxicity, and efficacy of 2-step, pretargeted radioimmunotherapy using a chimeric bispecific antibody and 131I-labeled bivalent hapten in a phase I optimization clinical trial. J Nucl Med 2006; 47:247-55.

45. Chatal JF, Campion L, Kraeber-Bodere F, Bardet S, Vuillez JP, Charbonnel B, et al. Survival improvement in patients with medullary thyroid carcinoma who undergo pretargeted 
anti-carcinoembryonic-antigen radioimmunotherapy: a collaborative study with the French Endocrine Tumor Group. J Clin Oncol 2006;24:1705-11.

46. Salavati A, Puranik A, Kulkarni HR, Budiawan H, Baum RP. Peptide receptor radionuclide therapy (PRRT) of medullary and nonmedullary thyroid cancer using radiolabeled somatostatin analogues. Semin Nucl Med 2016;46:215-24.

47. Iten F, Muller B, Schindler C, Rochlitz C, Oertli D, Macke $\mathrm{HR}$, et al. Response to [90Yttrium-DOTA]: TOC treatment is associated with long-term survival benefit in metastasized medullary thyroid cancer. A phase II clinical trial. Clin Cancer Res 2007;13(22 Pt 1):6696-702.

48. Beukhof CM, Brabander T, van Nederveen FH, van Velthuysen MF, de Rijke YB, Hofland LJ, et al. Peptide receptor radionuclide therapy in patients with medullary thyroid carcinoma: predictors and pitfalls. BMC Cancer 2019; 19:325. 